\title{
Does Final-Needle Aspiration Cytology Of The Breast Is Still An Accurate Diagnostic Technique for Breast Lumps?
}

\author{
Taha M M. Hassan ${ }^{(1)}$, Anil Mohan Rao. $\mathrm{S}^{(2)}$ \\ Department of Pathology, College of Medicine, BaniSweif University $(\text { Egypt })^{(I)}$, Faculty of Medicine \\ Northern Border University Saudi Arabia ${ }^{(2)}$
}

\begin{abstract}
:
Background: Fine-needle aspiration cytology (FNAC) is a simple, accurate, and safe procedure for the diagnosis of palpable breast lumps (PBLs), yet in certain situation there is a need for more definitive and more invasive techniques such as core needle biopsy $(C N B)$ to touch a definitive diagnosis. This procedure is performed instead of excisional or frozen section in some breast cancer units. The aim of this study was to analyze if FNAC is still an accurate procedure for diagnosis of PBLs as well as to correlate between FNAC and histopathological findings of CNB in PBLs.

Material and methods: An observational study was conducted on 66 patients presenting with PBLs. All these patients underwent outpatient FNAC. Core needle biopsy (CNB) was performed for the inadequate, suspicious, and malignant cases. Evaluation of all the prepared cytologic and histopathological slides was done.

Results: benign tumors are forming the major component of FNAC cases that seen, majority of themis fibroadenoma. Conversely 7 cases are cytologically malignant that proved histologically to be malignant. Three out of 6 cases that are cytologically highly suspicious are diagnosed malignanthistologically.

Conclusion: in spite of the simplicity, rapidity, and cost effectiveness of FNAC in the preoperative diagnosis of cancer, tissue diagnosis by CNB is highly recommended in selected cases for more confirmation.
\end{abstract}

Key Words: FNAC, Breast, PBLs, Carcinoma, Histopathology

\section{Introduction}

Fine-needle aspiration cytology (FNAC) is part of the triple assessment for the diagnosis of PBLs. It is an established, highly accurate method for diagnosing breast cancer (BCA) and has given rise to a reduction in the number of excision biopsies for benign breast disease (Mottahedeh et al., 2003). Additionally, FNAC forms part of the clinical workup of all patients with palpable lumps especially in the breast (Srilakshmi and Chavda 2013). In the recent years and in spite of the decline of the uses of FNAC particularly in many industrialized countries, this technique still plays a major role in the diagnosis of breast lumps whether palpable or non-palpable in other countries (Abdel-Hadi et al., 2010; David, 2009). In experienced hands, palpationguided FNAC is an excellent diagnostic tool. However, there is a movement toward using imaging guidance to target all masses (David 2008). Sometimes there is a limitation in the definitive diagnosis with FNAC alone, which in majority of these situation is linked to the inadequate material for cytological interpretation(Atamdede and Isaacs 1993, Collaco et al., 1999, Dominaguez et al., 1997, and Vertrani et al., 1992). Finally, despite the wide use of FNAC for the diagnosis of PBLs, it has not achieved improvement in the pre- surgical decision-making and management process for both the surgeon and the oncologist (Bdour et al.,2008).

\section{Material andMethods}

The present study was performed on 66 patients complaining of palpable breast lumps (PBLs) in a period of 3 years through November 2010 to October 2013. The attending patients were examined clinically in the surgical outpatient clinic, Arar central Hospital, KSA. Fine needle aspiration cytology was performed for all these patients under the supervision of the consultant surgeon. The procedure of aspiration was done by the use of 25-gauge syringes under ultrasongraphic guided techniques. The nodule (s) to be aspirated was/were identified; the overlying skin is cleansed with alcohol. After withdrawal of the syringe, one drop of aspirated material is forced into each of several labeled microscopic slides with the patient' name and hospital number. Frequently, 8 to 10 slides were used, as well as from 2 to 4 aspirates were performed for accurate cytological evaluation (Oertal 1996). The obtained material is immediately immersed in absolute alcohol for fixation which was sent for the department of pathology for slides preparation. Following the cytological examination, all suspicious, malignant and inadequate cases were subjected to other invasive manoeuvers as core needle biopsy (CNB).Then the obtained tissue is fixed in 10\% neutral puffered-formalin (Gattsuo et al., 2010). The smear slides were stained with Hematoxylin and Eosin (H\&E) stains, as well as air dried smears are subjected to DiffQuik for immediate evaluation (Ariga et al., 2002). The histological slides were prepared from the tissue specimens for paraffin-embedded formalin-fixed blocks then stained with H\&E. All slides were evaluated by 2 
experienced pathologists. All the examined FNAC cases were categorized according to standardized diagnostic categories in the form of 5 standard options includes: inadequate/insufficient, benign, atypical/indeterminate, suspicious of malignancy and malignant(National Breast Cancer Centre 2004). The adequacy of FNAC is dependent on multiple factors that include nature of the lesion, the available technology, and the experience and preference of the operator (Zakhour and Wells 1999). The smear is considered inadequate if there is hypocellularity, aspiration or smearing or staining errors (Zakhour and Wells 1999).

Study approved by ethical committee

\section{Statistical analysis}

The statistical analysis was undertaken using SPSS computer software (SPSS version 16 Microsoft windows). $\mathrm{Z}$ test was used for the comparison between two proportions. Results were considered to be statistically significant at $\mathrm{p}<0.05$.

\section{Results}

Cytologically, the inflammatory lesions are seen in 7 out of all cases (10.6\%) (Figure 1), benign FNACs cases representing the commonest lesions, that are found in 37 cases $(56.1 \%)$ (Figure 2), highly suspicious lesions are encountered in 6 cases $(9.1 \%$ ) (Figure 3), whereas malignant FNACs are detected in 7 $(10.6 \%)$ (Figure 4), and the unsatisfactory cases are representing 9 cases (13.6\%) (Table 1).

Table 1: cytological categories of all studying cases

\begin{tabular}{|l|c|c|}
\hline Categories of mammary lesions & Number of cases & \% of cases \\
\hline Inflammatory & 7 & 10.6 \\
Benignlesions & 37 & 56.1 \\
Highly suspicious lesions & 6 & 9.1 \\
Malignant lesions & 7 & 10.6 \\
Unsatisfactory & 9 & 13.6 \\
Total & 66 & 100 \\
\hline
\end{tabular}

Subsequent histopathological examination was performed and showed all the inflammatory FNACs were proved inflammatory (Figure 5), whereas 48 (72.7\%) out of all cases as benign lesions (Figure 6-8) and $18(27.3 \%)$ cases are malignant. Additionally 3 out of the 6 suspicious cytological cases are malignant histologically and 3 are benign. In the same theme, all malignant cases on cytology are diagnosed also as malignant on histology (Figure 9\&10), whereas 6 out of the unsatisfactory cytological cases are found to be benign tumors histologically, and the other 3 cases are malignant histologically (Table 2).

Table 2: FNACs diagnosis versus histopathological diagnosis of all studying cases

\begin{tabular}{|c|c|c|c|}
\hline \multirow{2}{*}{ FNACs } & \multirow{2}{*}{ \# of cases } & \multicolumn{2}{|c|}{ Histopathological diagnosis } \\
\hline & & Benign & Malignant \\
\hline Inflammatory & $7(10.6 \%)$ & 7 & 0 \\
\hline Benign lesions & $37(56.1 \%)$ & 32 & 5 \\
\hline Highly suspicious & $6(9.1 \%)$ & 3 & 3 \\
\hline Malignant lesions & $7(10.6 \%)$ & 0 & 7 \\
\hline Unsatisfactory & $9(13.6 \%)$ & 6 & 3 \\
\hline Total & 66 & $48(72.7 \%)$ & $18(27.3 \%)$ \\
\hline
\end{tabular}

Histologically, invasive duct carcinoma is the major subtype and representing 13 out of the 18 cases followed by mucinous carcinoma that encompassing 3 cases, whereas invasive lobular carcinoma is seen in 2 cases (Table 3). 
Does Final-Needle Aspiration Cytology Of The Braest Is Still An Accurate Diagnostic Technique For

Table 3: FNACs diagnosis and histopathological subtypes of malignant lesions in all studied cases $($ No. $=18)$

\begin{tabular}{|l|l|l|l|}
\hline FNACs & No. of cases & $\begin{array}{l}\text { histopathological } \\
\text { subtypes }\end{array}$ & No. of cases \\
\hline Benign lesions & 5 & $\begin{array}{l}\text { IDCa } \\
\text { Mucinous Ca } \\
\text { ILCa }\end{array}$ & 3 \\
& & IDCa & 1 \\
\hline Highly suspicious & 3 & $\begin{array}{l}\text { IDCa with comedo-type DCIS } \\
\text { Mucinous Ca } \\
\text { Pleomorphic ILCa }\end{array}$ & 3 \\
\hline Malignant & 7 & IDCa & 1 \\
& & Mucinous Ca & 1 \\
\hline Unsatisfactory & 3 & & 2 \\
& & 18 & 18 \\
\hline Total number & 18 & \\
\hline
\end{tabular}

IDCa; invasive duct carcinoma, ILCa; invasive lobular carcinoma

In concern to the cyto-histopathological correlation among the benign lesions, fibroadenoma represents the major category that is seen in 29 out of all benign cases (60.4\%), fibrocystic changes seen in 13 cases (27\%), intraductal papilloma in 2 cases (4.2\%), chronic abscess in 2 cases (4.2\%), and mastopathy found in 2 cases (4.2\%) (Table 4).

Table 4: Cyto-histopathological correlation of benign lesions (No.=37)

\begin{tabular}{|l|l|l|l|}
\hline FNACs diagnosis & No. of cases & Histological diagnosis & $\begin{array}{l}\text { No. } \\
\text { cases }\end{array}$ \\
\hline Benign lesions & 16 & $\begin{array}{l}\text { Fibroadenoma } \\
\text { Papilloma } \\
\text { Fibrocystic change (FCC) } \\
\text { Mastopathy }\end{array}$ & $\begin{array}{l}12 \\
2 \\
1\end{array}$ \\
\hline Fibroadenoma & & Fibroadenoma & 1 \\
& 12 & Lactating adenoma & 11 \\
& & FCC & 1 \\
\hline Cystic lesions & 7 & Epidermoid cyst & 5 \\
& & Fibroadenoma & 2 \\
\hline Fibroadenosis & 2 & FCC & 1 \\
\hline Total & & & 37 \\
\hline
\end{tabular}

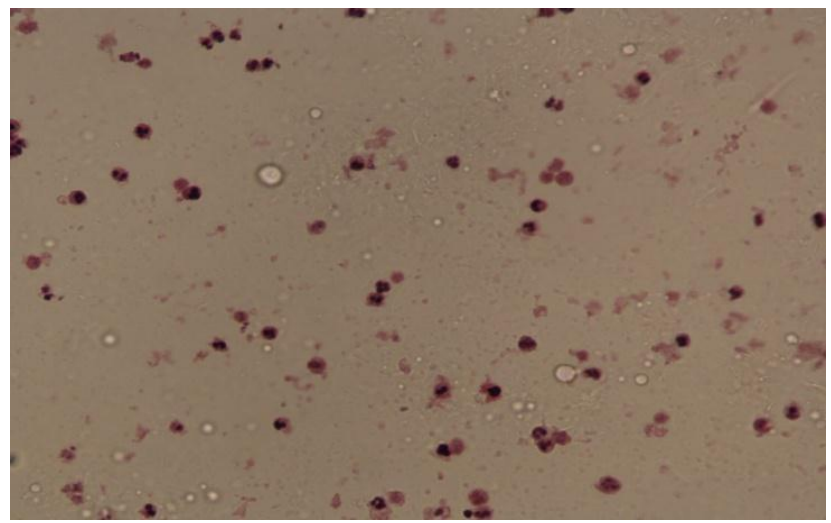

Figure 1: a case of inflammatory FNAC showing scattered inflammatory cells in a proteinaceous background (H\&E 100X) 


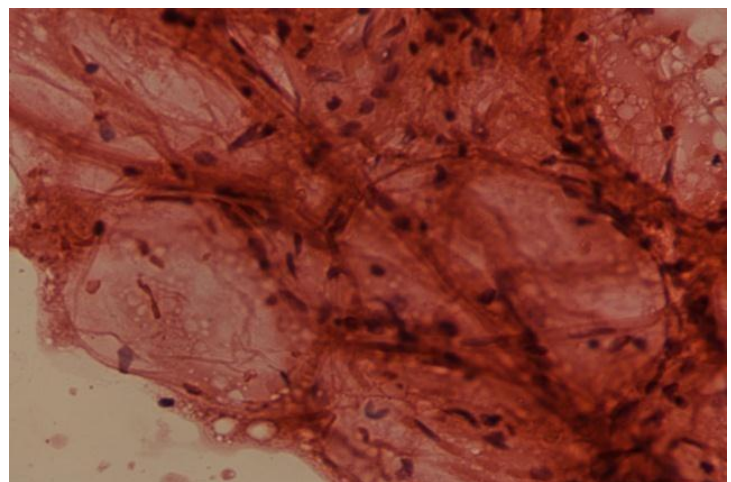

Figure 2: a case of benign FNAC showing stromal cells and occasional ductal cells (H\&E 100X)

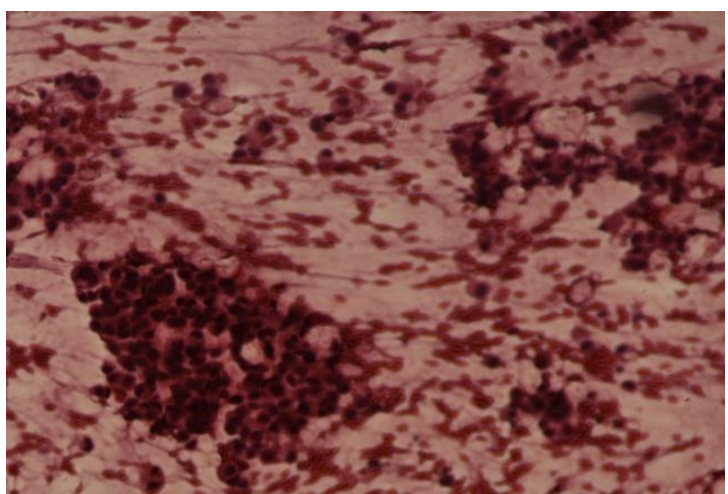

Figure 3: a case of highly suspicious FNAC showing discohesive clusters of epithelial cellswith hyperchromatic nuclei (H\&E100X)

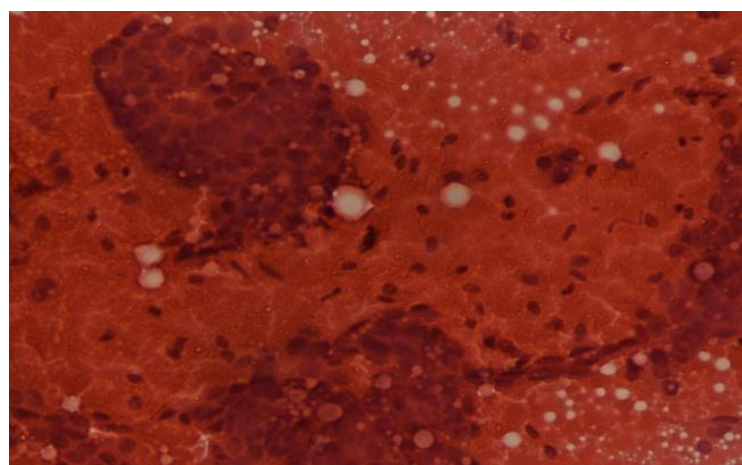

Figure 4: a case of malignant FNAC showing 3-dimentional sheets of malignant cells with pleomorphic hyperchromatic nuclei (H\&E200X)

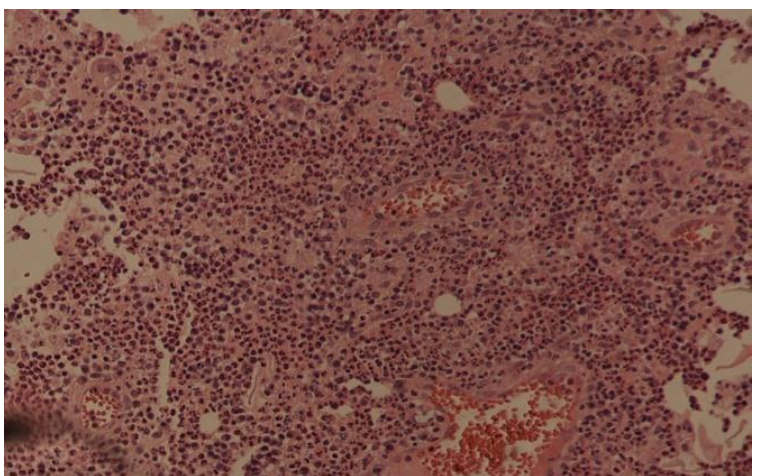

Figure 5: a case of core needle biopsy (CNB) showing neutrophilic infiltration with dilated congested vessels $(\mathrm{H} \& \mathrm{E} 100 \mathrm{X})$ 


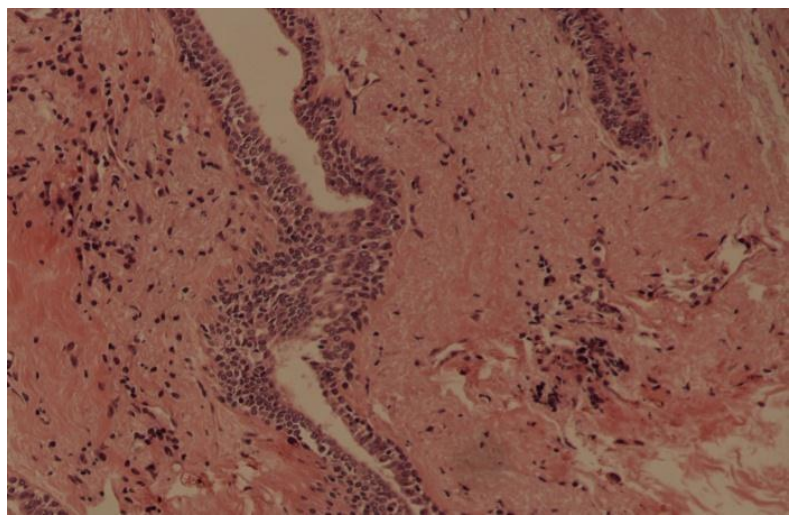

Figure 6: a case of CNB revealing proliferative mammary lesion with epithelial hyperplasia (H\&E 200X)

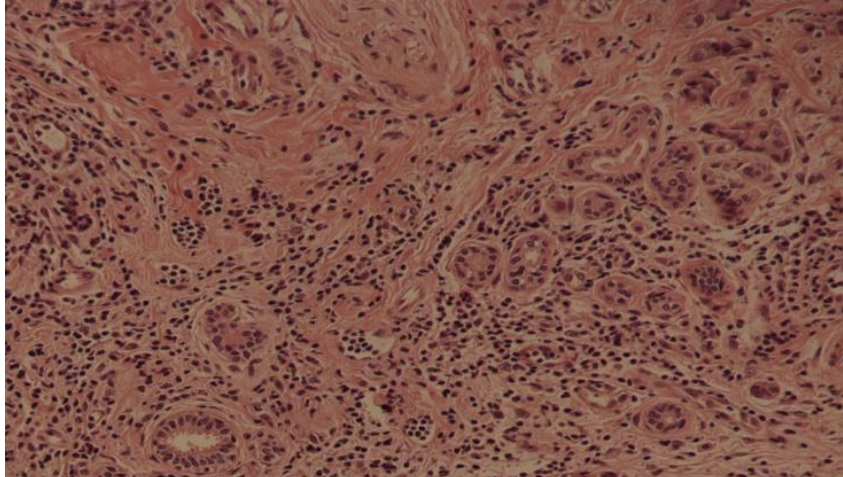

Figure 7: a case of CNB showing adenosis admixed with inflammatory cells in a fibrotic stroma (H\&E 100X)

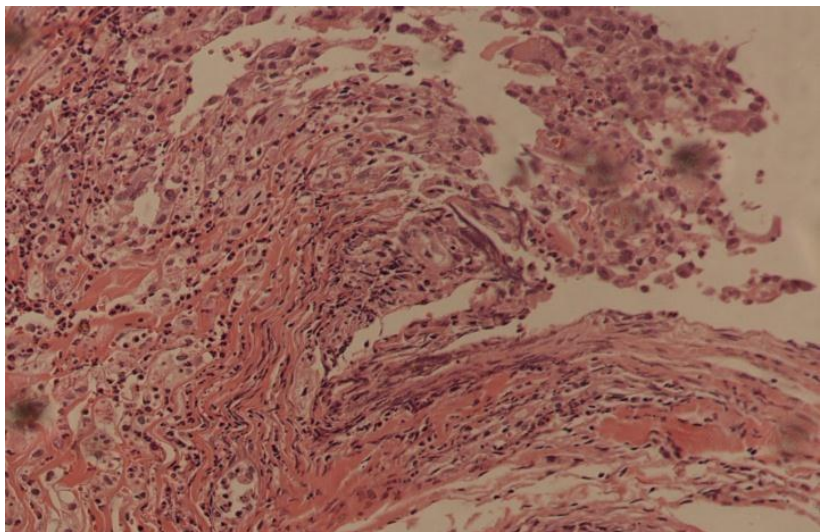

Figure 8: a case of CNB showing ruptured epidermal cyst with granulomatous inflammatory reaction (H\&E 100X)

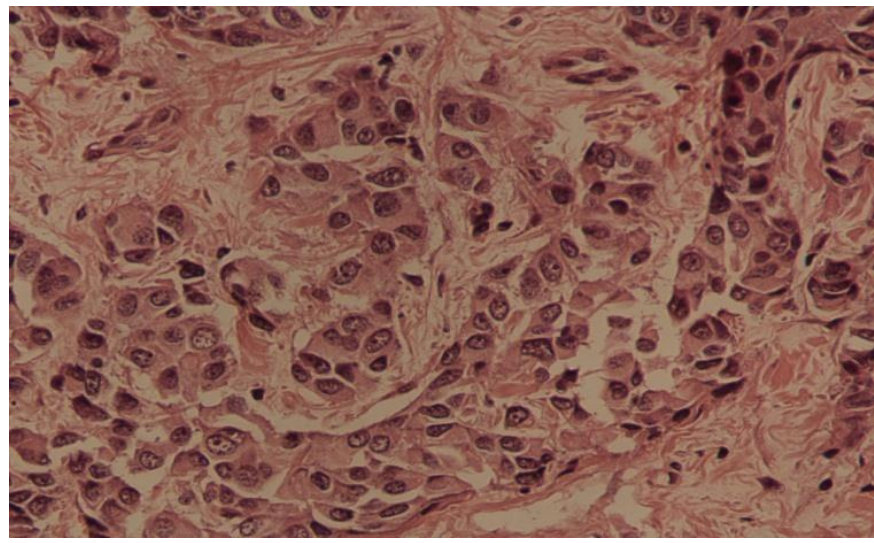

Figure 9: a case of CNB showing sheets of malignant cells with pleomorphic hyperchromatic nuclei admixed in desmoplastic stromal reaction (H\&E 200X) 


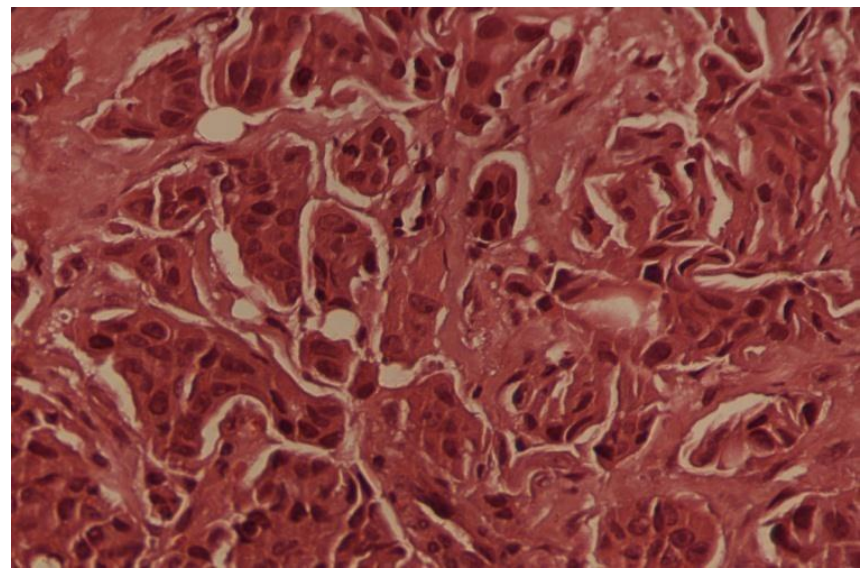

Figure 10: this case of CNB showing trabeculae of malignant cells with pleomorphic hyperchromatic nuclei $(H \& E$ 200X)

\section{Discussion}

Despite the evidence of FNAC's value in diagnosis as has been previously reported, in recent years, its use in the diagnosis of breast lesions has declined in many health services providers. Today the surgeons want to know the grade and subtype of breast cancer (Ahmed et al., 2009; Day et al., 2008). Currently, in high-resource countries, there is a trend away from FNACs of breast lesions and increased usage of core needle biopsy (CNB) as the first-line diagnostic method in many settings. However, this is not always for evidence-based reasons. Although the basis for this trend remains controversial, possible influencing factors include: difficulty in distinguishing in situ from invasive carcinoma and the lack of training in FNA sampling in many centers which has contributed to substandard results of FNAC and a resulting search for more reliable methods (Abdel-Hadi et al., 2010; Masood et al.,2003). Combined with poor financial reimbursement for the procedure, the aforementioned factors may contribute to decreased usage of breast FNAC in the USA, Canada, and the United Kingdom (Day et al., 2008; Nasuti et al.,2002).

Cytologically this study revealed inflammatory lesion of the breast observed in 7 cases (10.6\%), benign lesions detected in 37 cases $(56.1 \%)$ which representing the commonest lesions, highly suspicious lesions in 6 cases $(9.1 \%)$, malignancy in 7 cases $(10.6 \%)$, and the unsatisfactory cases are found in 9 cases $(13.6 \%)$. Many previous studies reported variable indices relating to benign FNACs in the total numbers of cases involved among them as O'Neil et al., (1997) found benign FNACs in $24 \%$ out of 697 cases, Ishikawa et al., (2007) in $47.6 \%$ out of 382 cases Rosa et al., (2011) in $60 \%$ out of 1583 of cases Feichter et al., (1997) in $68.1 \%$ out of 1003 cases whereas, Day et al., (2008) observed it in $77.5 \%$ out of 831 cases. A study performed in Egypt on 246 patients found that benign lesions were in near to suspicious FNACs and were detected in 31 cases for benign $(12.5 \%)$ and 28 for atypical / suspicious (11.4\%) whereas, 162 were malignant $(65.7 \%)$ that were representing the majority of cases (Abdel-Hadi et al., 2010).

In this study 9 cases are in the category of unsatisfactory FNACs and is mainly due to marked hypocellularity, yet histologically 3 cases among them are malignant. The limitations of FNACs interpretation are mainly due to poor cellularity of mammary tumors with fibrotic stroma, poor preservation and difficulty in cytological differentiation of atypical benign lesions and well-differentiated malignant neoplasms (Atamdede and Isaacs 1993, Collaco et al., 1999, Dominaguez et al., 1997, and Vertrani et al., 1992). Additionally, cytological discrepancies frequently encountered are inherent to FNAC of palpable breast lumps (PBLs) (Ariga et al., 2002). False negative FNAC of the breast are more common with invasive duct carcinoma than invasive lobular carcinoma (Boerner and Sneige 1998; Layfield and Dodd 1996).

Histopathologicallyfibroadenoma representing the major category that is seen in 29 out of all benign breast lesions (60.4\%), fibrocystic changes (FCC) in 13 cases (27\%), intraductal papilloma in 2 cases $(4.2 \%)$, chronic abscess in 2 cases (4.2\%), and mastopathy found in 2 cases $(4.2 \%)$. In reviewing the literature, The bulk of breast FNAC diagnoses are benign, accounting for 24-77.5\% of cases followed by FCC which showing a spectrum of histological features in which cystic changes represent a common finding (Mendoza et al., 2011).In the same theme fibroadenoma is the most solid breast lesions and its diagnosis is highly accurate and a study reported79.3\% predictive value out of 362 fibroadenoma aspirates with most diagnostic errors occurring in the older age group in addition to this, multiple fibroadenomas are seen in 15 to $20 \%$ of FNAC cases(LopezFerreret al., (1999); Zakhour and Wells 1999).

In this study core needle biopsy (CNB) was performed for all malignant, suspicious, benign, and the unsatisfactory cases that revealed invasive duct carcinoma (IDCa) is the major subtype of malignant cases and was representing 13 cases followed by mucinous carcinoma that encompassing 3 cases, whereas invasive 
lobular carcinoma is seen in 2 cases. A study performed on 200 patients with palpable breast lumps reported out of 65 benign FNAC cases, 5 cases were found malignant on histopathology, out of 5 suspicious cases on cytology, 3 cases were found malignant on histopathology, and all malignant FNACs cases were proved on histology as cancer in agreement with our findings (Ahmed et al., 2009).

Although some authors consider that FNAC might be abandoned in favor of CNB (Litherland 2001; Shannon et al., 2001), the opinion that CNB does not replace FNAC as a first-line diagnostic modality for a breast lesion is evidenced by that CNB is not able to establish the diagnosis of malignancy revealed by FNAC in many results as Barra Adeet al., (2008) reported CNB was not able to establish the diagnosis for malignancy revealed by FNAC in 14 cases. Like FNAC, the accuracy of CNB in diagnosing breast cancer also varies in the literature. Dillon et al., (2005) reported false negative rates ranging from 0 to $12 \%$ for ultrasound guided CNB. Comparisons of sensitivity and specificity rates between FNAC and CNB have on occasion found that FNA has a higher accuracy rate in diagnosing breast cancers. Ballo and Sneige (1996) reported sensitivity and specificity of $97.5 \%$ and $100 \%$, respectively, in FNA compared with $90 \%$ and $100 \%$, respectively in CNB. Also they reported that sampling errors were the only cause of false-negative diagnoses with CNB.

Conflict of interest

\section{Acknowledgment}

There is no conflict of interest

\section{References}

[1]. Mottahedeh M, Rashid MH, Gateley CA. Final diagnoses followingC3 (atypical, probably benign) breast cytology. Breast 2003;12:276279 .

[2]. Srilakshmi HP and Chavda J. A study of cyto-histological correlation of breast lesions. NJIRM 2013; 4(2) : 54-56.

[3]. David L. Value of cytopathologist-performed ultrasound-guided fine needle aspiration as a screening test for ultrasound-guided coreneedle biopsy in non-palpable breast masses. DiagnCytopathol 2009;37:262-269.

[4]. Abdel-Hadi M, Abdel-Hamid GF, Abdel-Razek N, Fawzy RK. Should Fine-Needle Aspiration Cytology be the First Choice Diagnostic Modality for Assessment of All Non palpable Breast Lesions? The Experience of a Breast Cancer Screening Center in Alexandria, Egypt. Diagnostic Cytopathology 2010, Vol 38, No 12:880-889.

[5]. David L. Cytopathologist-performed ultrasound-guided fine-needle aspiration and core-needle biopsy: A prospective study of 500 consecutive cases. Diagnostic Cytopathology 2008, vol. 36, issue5, May 2008:

[6]. Atamdede FI and Isaacs JH. The role of fine needle aspiration in the diagnosis of breast lesions.GynecolOncol 1993; 50(2): 159- 63.

[7]. Collaco LM, de Lima RS, Werner B, and Torres LF. Value of fine needle aspiration in the diagnosis of breast lesions.ActaCytol 1999; 43(4):587-92.

[8]. Dominguez F, Riera JR, and Tojo sand Junco P. Fine needle aspiration of breast masses: An analysis of 1398 patients in a community hospital. ActaCytol 1997; 41(2):341-47.

[9]. VertraniFI, Fulciniti F, DiBenedetto G, Zeppa P, Tronocone G, Bascanio A, Rossa GD, and PalmbiniL. Fine-needle aspiration biopsyofbreastmasses:Anadditionalexperiencewith1553cases(1985-1988)andmeta-analysis. Cancer1992;69(3):736-40.

[10]. Bdour M, Hourani S, Mefelh W, Shabatat A, Karadsheh S, Nawaiseh O, Ebous A. Comparison between fine needle aspiration cytologyandtru-cutneedlebiopsyinthediagnosisofbreastcancer. J ofSurgPakistan (Inter.)2008,13(1):1-3.

[11]. Oertel YC., Fine needle aspiration and diagnosis of thyroid cancer. EndocrinolMetabClin North Am 1996; 25: 69-71.

[12]. Gattuso, Reddy, David, Sptiz, and Haber. Differentitial diagnosis in surgical pathology.Saunders Elsevier; 2010; $2^{\text {nd }}$ Ed. Vol. 1 P. 2

[13].Ariga R, Bloom K, Reddy VB, Kluskens L, Franscecatti D, Dowlat K, Siziopikuo P, Gatuso P. Fine needle aspiration of clinically suspicious breast masses with Histopathologic correlation. Am J Surg 2002; 184(5): 410-413.

[14]. National Breast Cancer Centre 2004. Breast fine needle aspiration cytology and core biopsy: a guide for practice, $1{ }^{\text {st }}$ Ed. P. 42; ISBN Print: 1741270367

[15]. ZakhourH and Wells C. Diagnostic Cytopathology of the Breast, Churchill Livingstone, London, UK, 1999.

[16]. Ahmed HG, Ali AS, Almobarak AO. Utility of Fine-Needle Aspiration As a Diagnostic Technique in Breast Lumps. Cytopathol 2009;37:881-884

[17]. Day C, Moatamed N, Fimbres AM, Salami N, Lim S, Apple SK. A retrospective study of the diagnostic accuracy of fine-needle aspiration for breast lesions and implications for future use.DiagnCytopathol2008;36:855-860.

[18]. Masood S, LoyaA, Khalbuss W. Is core needle biopsy superior to fine-needle aspiration biopsy in the diagnosis of papillary breast lesions? DiagnCytopathol2003;28:329-334.

[19]. Nasuti JF, Gupta PK, Baloch ZW. Diagnostic value and cost effectiveness of on-site evaluation of fine-needle aspiration specimens: Review of 5,688 cases. DiagnCytopathol2002:27:1-4

[20]. O'Neil S, Castelli M, Gattuso P, KluskensL,MadsenK,andAranha G. Fine-needle aspiration of 697 palpable breast lesions with histopathologic correlation. Surgery 1997; Vol. 122, No.4:824-828.

[21]. Ishikawa $\mathrm{H}$, Hamaguchi $\mathrm{Y}$, Tanabe $\mathrm{M}$, et al. False-positive and false-negative cases of fine-needle aspiration cytology for palpable breast lesions. Breast cancer 2007; Vol. 14, No. 4:388-392.

[22]. Rosa M, Mohammadi A, and Masood S. The value of fine needle aspiration biopsy in the diagnosis and prognostic assessment of palpable breast lesions.DiagCytopathol 2011; Vol. 39, No.6,

[23]. Feichter GE, Haberth"ur F, Gobat S, and Dalquen P. Breast cytology: statistical analysis and cyto-histologic correlations. ActaCytologica 1997; Vol. 41, No. 2:327-332.

[24]. Boerner S, Sneige N. Specimen adequacy and false-negative diagnosis in fine-needle aspirates of palbaple breast masses. Cancer 1998; 84(6):344-348

[25]. Layfield LJ, Dodd LG. Cytologically low grade malignancies: an important iterpretative pitfall responsible for false negative diagnosis in fine-needle aspiration of the breast. DiagnCytopathol 1996; 15(3):250-259.

[26]. Mendoza P,LacambraM,TanPH,Tse GM. Fine Needle Aspiration Cytology of the Breast: The Nonmalignant Categories. Pathol Res Inter; 2011: 1-8. 
[27]. L'opez-Ferrer P, Jim'enez-Heffernan JA, Vicandi B, Ortega L, Viguer JM. Fine needle aspiration cytology of breast fibroadenoma: a cytohistologic correlation study of 405 Cases. ActaCytologica 1999; Vol. 43, No. 4:579-586.

[28]. Litherland JC. Should fine-needle aspiration cytology in breast assessment be abandoned? ClinRadiol2001;57:81-84

[29]. Shannon J, Douglas-Jones AG, Dallimore NS. Conversion to core biopsy in preoperative diagnosis of breast lesions: Is it justified by results? J ClinPathol2001;54:762-765.

[30]. Barra Ade A, Gobbi H, de L Rezende CA, et al. A comparison of aspiration cytology and core needle biopsy according to tumor size of suspicious breast lesions.DiagnCytopathol2008;36:26-31.

[31]. Dillon MF, Hill AD, Quinn CM, O’Doherty A, McDermott EW, O’Higgins N. The accuracy of ultrasound, stereotactic, and clinical core biopsies in the diagnosis of breast cancer, with an analysis of false-negative cases. Ann Surg 2005;242:701-707.

[32]. Ballo MS andSneige N. Can core needle biopsy replace fine-needle aspiration cytology in the diagnosis of palpable breast carcinoma. A comparative study of 124 women Cancer1996;78:773-777. 